\title{
Acupuncture: An Important Modality In Non-Pharmacological Pain Management
}

\author{
Yemeng Chen* \\ New York College of Traditional Chinese Medicine, USA
}

Submission: November 06, 2017; Published: January 23, 2018

*Corresponding author: Yemeng Chen, Ph.D., New York College of Traditional Chinese Medicine, Mineola, NY 11501, USA, Tel: 516-739-1545 x 208; Fax: 516-873-9622; Email: yemeng.chen@nyctcm.edu

\begin{abstract}
In the current crisis of abuse and misuse of prescription opioids, acupuncture is considered as an important non-pharmacological pain management modality. Acupuncture not only takes care of pain symptoms but also deals with whole body balance. It is a drug-free, cost-effective, and harmless therapy with a tremendous number of indications. Scientific data shows favorable outcomes towards pain management and also for drug addiction. Advanced acupuncture therapies have provided more opportunities for pain management. The mainstream medical field has accepted acupuncture in a favorable way, but the profession should also enhance their knowledge and competencies in order to provide a higher standard of acupuncture treatment.
\end{abstract}

Keywords: Acupuncture; Opioids crisis; Non-Pharmacological treatment; Pain management

\section{Introduction}

Abuse and misuse of prescription opioids has became a social crisis in the United States, such that overdose deaths from prescription opioids (including nonmethadone synthetics) have gone up in the past fifteen years to about 20,000 in 2015 [1]. In response to this, nonpharmacological treatments, including acupuncture, for pain management have been strongly suggested by different national authorities, such as the FDA and National Academies of Sciences, Engineering, and Medicine [1,2].

As one of the non-pharmacological modalities, acupuncture has demonstrated its efficacy in clinical practice for a significant amount of indications including pain management. This three-thousand-year-old therapy provides drug-free, costeffective, and harmless treatment which has recently been applied clinically worldwide. Compared with the other natural modalities, acupuncture not only takes care of some symptoms, like pain, but also deals with whole body balance according to its philosophy. Since the 1970's, acupuncture analgesic studies have been conducted and neurophysiologic mechanisms such as Gate Theory and $\beta$-endorphin type of natural pain-killer release activation have been discovered. Although some clinical trials have raised the issue of the placebo effect of acupuncture, a large scale meta-analysis (nearly 18,000 randomized patients on high quality trials) has demonstrated acupuncture effectiveness in non-specific musculoskeletal pain (back and neck), chronic headache, shoulder pain and osteoarthritis [3]. It also concluded that acupuncture is effective for the treatment of chronic pain and is therefore a reasonable referral option. Significant differences between true and sham acupuncture indicate that acupuncture is more than a placebo. Recent newly published articles also showed acupuncture can reduce pain or opioid consumption after total knee arthroplasty in a meta analysis of 39 trials involving 2391 patients among the data of 5509 trials from January 1946 to April 2016 [4]

Besides effectively dealing with pain management, acupuncture works for drug addiction. Some reports showed the efficacy in drug addiction also for opiate addiction $[5,6]$.

Other than traditional acupuncture needles and moxibustion-leaves of mugwort processed and used as heat stimulation towards acupoints--there are tremendous advancements at present, including different stimulating systems like auricular, scalp, wrist-ankle, navel, and abdominal acupuncture in addition to regular body acupuncture, different stimulating tools such as seven-star needles and thee-edged needles, and different stimulating instruments like electro-acupuncture. The latter one has more efficacy in treating neurological issues and severe pain syndromes. A new therapy of dry-needling has recently emerged, which is a part of acupuncture using the same needles as acupuncturists use. For over a thousand years of practice, methods of tender spot palpation and then needling as so called "A-Shi Point" acupuncture have been effectively applied 
for musculoskeletal disorders. 20 or even 50 hours of training to perform dry-needling by other healthcare practitioners such as physical therapists are not appropriate and not legal also. Acupuncturists are well trained in needle practice with a minimum 2000 hours training needed to acquire an acupuncture license. In the new entry-level professional doctorate program, the curriculum has its required competencies of advanced diagnosis, system-based medicine, and professional development based on current master's program competencies. So the philosophy and concept originated from ancient China, and the practice is in modern society.

The mainstream medical field has gradually accepted acupuncture as one kind of integrative medicine therapy. More favorable news has come this year. The American College of Physicians published its Clinical Practice Guidelines for lower back pain treatment in February 2017 in which acupuncture was recommended as one of the first line treatments for all acute, subacute and chronic lower back pain [7]. The Joint Commission (JC), the largest U.S. accreditation body for health care facilities, announced a revision of hospital performance measures for pain management that includes the requirement of providing nonpharmacological pain treatment modalities, effective January 1, 2018 [8]. In this specific standard (LD.04.03.13), JC provides examples of evidence-based nonpharmacological strategies that could be provided, including acupuncture therapy, osteopathic manipulation, and massage therapy. So it can be predicted that acupuncture therapy will be available in the near future as one of the important pain management modalities in hospitals throughout the US.

Under these special circumstances, profession-wide enhancement of knowledge and competencies--especially concepts about integrative medicine--is deemed most important, including establishing protocols of acupuncture delivery in the healthcare system, creating treatment plan standards, and training other healthcare providers and patients about acupuncture. Higher quality of scientific-based clinical trials and laboratory studies are expected to prove the efficacy of acupuncture and to explain the mechanism of action and appropriate use.

For acupuncture professionals, this is indeed a great opportunity to promote the important role of integrative medicine during this opioid crisis. Acupuncture is truly beneficial for those patients and for society at large.

\section{Conclusion}

It is foreseeable that the acupuncture profession will expand its practice significantly in the near future.

\section{References}

1. Bonnie RJ, Ford MA, Phillips JK (2017) Pain Management and the Opioid Epidemic: Balancing Societal and Individual Benefits and Risks of Prescription Opioid Use. The National Academies Press, Washington, USA.

2. Thielking M (2017) FDA Proposes that doctors learn about acupuncture for pain management. STAT.

3. Vickers AJ, Cronin AM, Maschino AC, Lewith G, MacPherson H, et al (2012) Acupuncture for chronic pain: individual patient data metaanalysis. Arch Interm Med 172(19): 1444-1453.

4. Tedesco D, Gori D, Desai KR, Asch S, Carroll IR, et al. (2017) Drug-Free Interventions to Reduce Pain or Opioid Consumption After Total Knee Arthroplasty: A Systematic Review and Meta-analysis. JAMA Surg 152(10): e172872.

5. Cui CL, Wu LZ, Luo F (2008) Acupuncture for the treatment of drug addiction. Neurochem Res 33(10): 2013-2022.

6. Lin JG, Chan YY, Chen YH (2012) Acupuncture for the Treatment of Opiate Addiction. Evid Based Complement Alternat Med 2012: 739045.

7. Qaseem A, Wilt TJ, McLean RM, Forciea MA, Clinical Guidelines Committee of the American College of Physicians (2017) Noninvasive Treatments for Acute, Subacute, and Chronic Low Back Pain: A Clinical Practice Guideline From the American College of Physicians. Ann Intern Med 166(7): 514-530.

8. The Joint Commission (2017) Joint Commission Enhances Pain Assessment and Management Requirements for Accredited Hospitals. The Joint Commission Perspectives (The Official Newsletter of The Joint Commission 37(7): 1 .

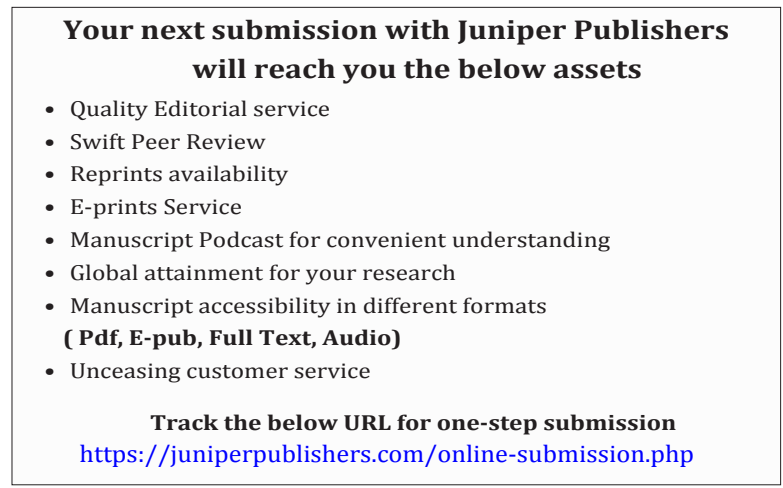

